\title{
Duloxetine in Affective Disorders: a Naturalistic Study on Psychiatric and Medical Comorbidity, Use in Association and Tolerability Across Different Age Groups
}

\author{
Bernardo Dell’Osso*, Giulia Camuri, Cristina Dobrea, Massimiliano Buoli, Marta Serati and A. \\ Carlo Altamura
}

\author{
Department of Psychiatry, University of Milan; Fondazione IRCCS Ca' Granda, Ospedale Maggiore Policlinico, Via \\ Francesco Sforza, 20122 Milano, Italy
}

\begin{abstract}
Objective: Duloxetine, a selective serotonin and norepinephrine reuptake inhibitor (SNRI), is currently approved in many countries for the treatment of Major Depressive Disorder (MDD) and Generalized Anxiety Disorder (GAD). The present naturalistic study was aimed to investigate tolerability of Duloxetine in a sample of patients with affective disorders and psychiatric/medical comorbidity, comparing tolerability in monotherapy versus polytherapy and across different age groups. Methods: The sample included 165 patients, affected by anxiety and/or mood disorders with or without comorbidity, who had been taken Duloxetine for at least 1 month. Sample variables were collected through a retrospective chart review. Results: Most common primary diagnoses were MDD (49.1\%), Bipolar Disorder (BD) (15.7 $\%)$ and GAD (5.5\%). The $40 \%$ of the sample had psychiatric comorbidity: in particular, anxiety disorders (15.8 \%) (GAD $7.9 \%$, Panic Disorder -PD- 7.3\%) and personality disorders $(9.1 \%)$ as the most frequent ones. With respect to medical comorbidities ( $68 \%$ of the sample), hypertension $(12.1 \%)$ and diabetes $(7.3 \%)$ were the most common ones. Mean duration of treatment and dosage of Duloxetine were, respectively, 11 months $( \pm 9.1)$ and $70 \mathrm{mg} /$ day $( \pm 28.6)$. The $68 \%$ of the sample received Duloxetine in association with other drugs. Minor side-effects, in particular drowsiness and gastrointestinal problems, were reported by $15 \%$ of the sample. No difference in terms of tolerability across distinct groups, divided on the basis of mono- vs polytherapy as well as of different age, was found. Conclusion: Duloxetine, mostly administered in patients with affective disorders with psychiatric/ medical comorbidity and in association with other drugs, appeared to be well tolerated, showing limited rates of side effects of mild intensity. Further naturalistic studies are warranted to confirm present results.
\end{abstract}

Keywords: Affective disorders, Duloxetine, medical comorbidity, psychiatric comorbidity, serotonin norepinephrine reuptake inhibitors, tolerability.

\section{INTRODUCTION}

Duloxetine, along with Venlafaxine, Desvenlafaxine and Milnacipran, is a relatively new compound belonging to the class of the SNRIs [1]. These agents present a dual mechanism of action, blocking selectively the synaptic reuptake of both serotonin (5-HT) and norepinephrine (NE) in the central nervous system [2]. Duloxetine, in particular, has been firstly approved by the Committee for Medicinal Products for $\mathrm{Hu}-$ man Use (CHMP) and, then, by several other global regulatory bodies such as Food and Drugs Administration (FDA), for the treatment of neuropathic pain, stress urinary incontinence, fibromyalgia, MDD and, more recently, GAD [3]. In Italy, approved indications for the prescription of this drug include MDD, GAD and neuropathic pain.

Duloxetine shows a linear kinetics at the therapeutic dosages (60-120 mg/day), has an oral bioavailability ranging from $32 \%$ to $80 \%$, a mean elimination half-life of about 12 hours and a median time of adsorption of 2 hours [4]. The

\footnotetext{
*Address correspondence to this author at the Department of Psychiatry, Università degli Studi di Milano, Fondazione IRCCS Ca' Granda, Ospedale Maggiore Policlinico, Via F. Sforza 35, 20122 Milano, Italy; Tel: 02-55035994; Fax: 02-50320310; E-mail: bernardo.dellosso@policlinico.mi.it
}

compound is highly bound to plasma proteins (96\%), extensively distributed throughout tissues and metabolized by cytochrome P450 isoenzymes 1A2 and 2D6 (which is also inhibited by the molecule) to inactive metabolites in the liver $[5,6]$. The fact that this molecule is extensively metabolized by cytochrome P450 implies that caution should be taken when co-administered with substrates of the isoenzyme such as tricyclics antidepressants, phenotiazine and type $1 \mathrm{C}$ antiarrhythmics $[7,8]$.

Duloxetine is generally safe and well tolerated: nausea, headache, dry mouth, insomnia, fatigue, constipation, diarrhea, dizziness, increased sweating, sexual dysfunction and decreased appetite are the most common side-effects reported by patients [9]. Recently, cases of Duloxetine-induced liver injury have also been observed in patients with preexisting liver disease or chronic alcohol use [10].

Duloxetine is considered an effective and safe compound for the treatment of MDD and GAD. Recent studies have also observed that Duloxetine represents an effective switch strategy for the treatment of SSRI-resistant Major Depression [11]. Different clinical trials - often conducted under open conditions - have investigated the efficacy and safety of Duloxetine in MDD and GAD. From this perspective, a re- 
cent study has shown that the switch from SSRI/SNRI to Duloxetine was effective in patients affected by MDD and painful physical symptoms. In particular, improvements in clinical measures of mood, pain and anxiety were accompanied by clinically meaningful functional improvements [12].

Another study conducted by Pitchot and colleagues, on a sample of 29 treatment-resistant depressed patients, has shown a significant decrease in the Clinical Global Impression scale scores after treatment with Duloxetine (dosages between 60 and $120 \mathrm{mg} /$ day). Remission was achieved by the $48 \%$ of the patients and tolerability profile was favourable, supporting the potential benefit of Duloxetine for treatment-resistant depressed patients, also in relation to remission rates [13]. Moreover, the dosage of $60 \mathrm{mg} / \mathrm{day}$ of Duloxetine was found to be effective in preventing depressive recurrences [14].

With respect to GAD, a recent meta-analysis of 27 randomized controlled trials, assessing efficacy of different licensed drug treatments, found that Duloxetine, Escitalopram and Pregabalin might offer some advantages in terms of response, remission and tolerability, respectively, over Venlafaxine and Paroxetine [15]. Furthermore, a recent article has analyzed results from 3 clinical trials finding that Duloxetine was efficacious in the short- and long-term treatment of painful physical symptoms (PPS) associated to GAD. Of note, the lack of response/remission to GAD symptoms was associated to a less robust improvement in PPS [16].

With respect to compliance to treatment, another recent study has found that Duloxetine showed higher adherence and persistence rate, as well as longer duration of therapy, compared to Venlafaxine XR and Escitalopram [17].

Even though Duloxetine' efficacy and tolerability for the treatment of affective disorders is well-established [18], little is known about its tolerability in naturalistic setting when used in poly-therapy and in the elderly. In this regard, Wise and co-workers have recently evaluated safety and tolerability of this compound in a subgroup of MDD patients aged between 65 and 89, comparing subjects with and without medical comorbidities (e.g., vascular disease, diabetes, arthritis), finding that Duloxetine $60 \mathrm{mg} /$ day was associated with greater improvement on cognition and depressive symptoms. In particular, efficacy and tolerability were not affected by the comorbidity status, supporting the use of this molecule in elderly patients as well [19].

The aim of the present study was, therefore, to assess the tolerability of Duloxetine in patients with affective disorders and psychiatric/medical comorbidity, comparing tolerability in monotherapy versus polytherapy and across different age groups.

\section{MATERIAL AND METHODS}

Eligible subjects were adult outpatients with affective disorders (i.e., any anxiety or mood disorder), of both male and female, with no limitation on age, who were taking Duloxetine in mono- or poly-therapy for at least 1 month. Monotherapy was intended as Duloxetine administered as sole agent while poly-therapy to augmentative Duloxetine used in add-on regimen to a pre-existing treatment.
In order to be included in the present study, patients had to show a good compliance to treatment assessed through monthly clinical evaluations and pill count. Moreover, when patients were on polytherapy, the associated therapy had to be maintained relatively stable for at least 1 month before the augmentation of Duloxetine, with no change except for the introduction of Duloxetine, in order to minimize confounding factors in the assessment of side-effects. With respect to mono- vs poly-therapy, we considered the association of Duloxetine with benzodiazepines as monotherapy, while all other psychopharmacological association as polytherapies. Informed written consent was obtained from all patients who gave their approval to participate into the study and for having their clinical charts reviewed for research purposes. An additional specific consent form was obtained for patients who received off-label Duloxetine.

The sample consisted of adult outpatients attending the Mood Disorder Clinic and related Community Services of the Department of Psychiatry of the University of Milan. Patients' primary diagnosis had to be of mood and/or anxiety disorders, according to the DSM-IV-TR criteria [20]. If a comorbid condition was present, the affective disorder had to be the primary disorder, that is the one providing the motivation to seek treatment and causing the most significant distress. Diagnoses were performed by trained psychiatrists using the Structured Clinical Interview for DSM-IV for Axis I and II disorders (SCID I and II) [21, 22]. Moreover, the diagnostic evaluation was completed by a psychometric assessment, using the Hamilton Rating Scale for Depression (HAM-D) [23], Hamilton Anxiety Scale (HAM-A) [24], Montgomery-Asberg Rating Scale for Depression (MADRS) [25], Young Mania Rating Scale (YMRS) [26] and the Clinical Global Impression (CGI) [27], in order to assess the overall clinical severity of the primary diagnosis and eventual comorbid conditions. Demographic and clinical data were collected through retrospective consultation of clinical charts, including age, gender, age at onset, family history for mental disorders, cross-sectional psychiatric and medical comorbidities, mean dosages of Duloxetine, associated therapy, side-effects and adverse events. An adverse event (AE) was defined as any unfavorable and unintended sign including an abnormal laboratory finding, symptom or disease associated with the use of the drug that occurs during the course of the study. Safety and tolerability were assessed using spontaneously reported events and rates of discontinuation for adverse events. Control visits after the introduction of Duloxetine were performed within the first following month.

The main demographic and clinical variables were tabulated and standard descriptive statistics produced. Continuous variables were compared using one-way analyses of variance (ANOVAs) while and $\chi^{2}$ test was used for comparing categorical ones. Post-hoc tests (Bonferroni) were additionally performed to assess the differences among groups (e.g.: monotherapy vs politherapy, $<$ or $\geq 65$ years). For all the analyses, the level of statistical significance was set at 0.05 and not adjusted.

\section{RESULTS}

From an original sample of 188 patients, 23 subjects were excluded from the analyses for not having satisfied 

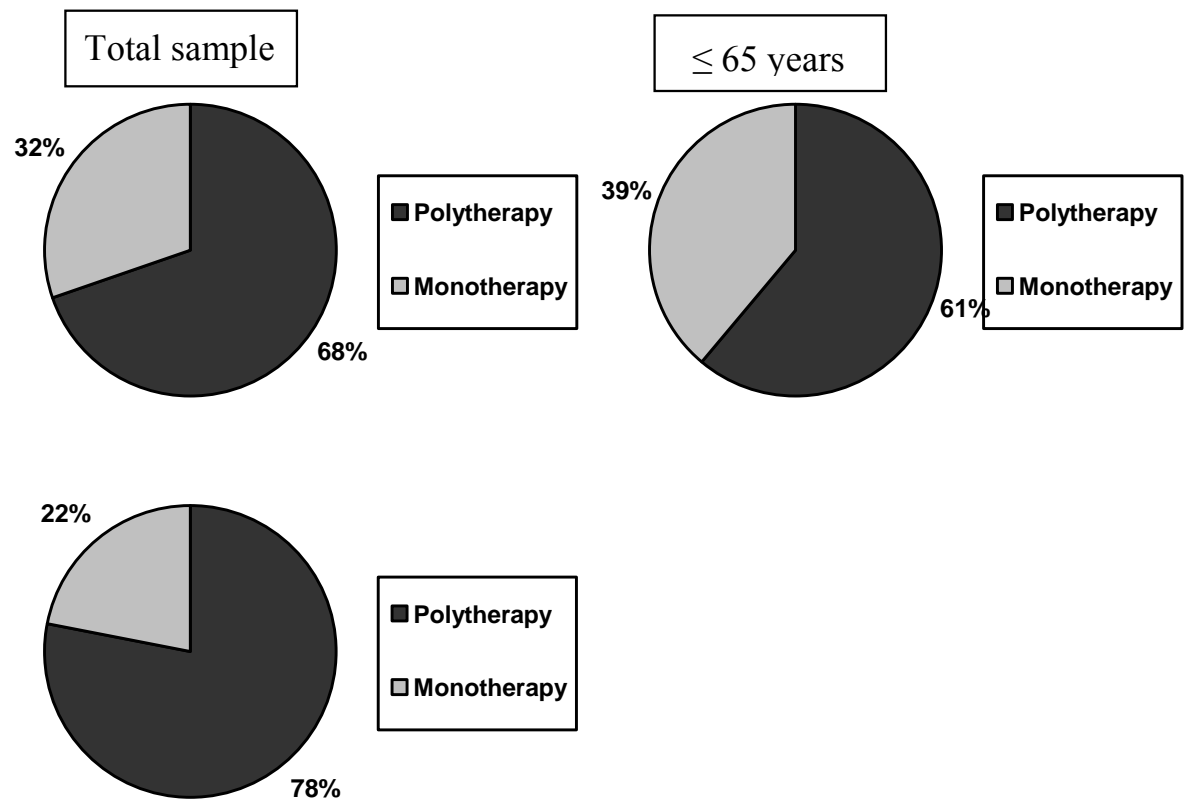

$$
>65 \text { years }
$$

Fig. (1). Polytherapy vs monotherapy in the total sample and accros different age groups.



Fig. (2). Side effects distribution in the overall sample

inclusion criteria (10 patients for having non-affective primary diagnosis [e.g., psychotic disorders, eating disorders, substance use disorders and personality disorders], 4 patients for Duloxetine-associated therapy major changes and 9 were lost to follow-up). The final sample comprised 165 subjects, 111 of whom females $(67.3 \%)$ and 54 males $(32.7 \%)$. MDD was the most common primary diagnosis $(n=81 ; 49.1 \%)$, followed by $\mathrm{BD}(\mathrm{n}=26 ; 15.7 \%)$, GAD $(\mathrm{n}=9 ; 5.5 \%)$ and Adjustment Disorder $(n=9 ; 5.5 \%)$. Nearly half of the sample $(\mathrm{n}=66 ; 40 \%)$ presented psychiatric comorbidity: in particular, anxiety disorders $(\mathrm{n}=26 ; 15.8 \%)$, with GAD $(\mathrm{n}=$ $13 ; 7.9 \%)$ and $\mathrm{PD}(\mathrm{n}=12 ; 7.3 \%)$ as the most frequent ones, and personality disorders $(\mathrm{n}=15 ; 9.1 \%)$ were the most common co-occurring conditions. Approximately 68\% (n = 112) of the sample had medical comorbidity with hypertension $(n=20 ; 12.1 \%)$ and diabetes $(n=11 ; 7.3 \%)$, being the most common diseases.
Mean duration and dosage of Duloxetine treatment were, respectively, 11 months $( \pm 9.1)$ and $70 \mathrm{mg} /$ day $( \pm 28.6)$. Of note, in the majority of the sample $(\mathrm{n}=112 ; 68 \%)$, Duloxetine was administered in association with other drugs. In particular, rates of co-administration with atypical antipsychotics were $13.3 \%, 11 \%$ with mood stabilizers, 15.1 $\%$ with atypical antipsychotics plus mood stabilizers and $9.1 \%$ for SSRIs (Fig. 1). Finally, more than half of the patients $(n=99 ; 60 \%)$ were receiving benzodiazepines.

No patients withdrew Duloxetine for AEs or side-effects. Side-effects were experienced by $15 \%(n=25)$ of the sample and were reported to be transient and mild in severity, with sedation $(\mathrm{n}=12 ; 7.3 \%)$ and gastrointestinal symptoms $(\mathrm{n}=$ $6 ; 3.6 \%$ ), such as nausea and constipation, being the most frequent ones (Fig. 2). No patient in the BD subgroup developed manic/hypomanic switch. 
Table 1. Main Demographic and Clinical Variables of the Study Sample

\begin{tabular}{|c|c|c|}
\hline \multicolumn{2}{|r|}{ Variables } & Mean/Percentages \\
\hline \multirow{2}{*}{ Gender } & Males & $32.7 \%$ \\
\hline & Females & $67.3 \%$ \\
\hline \multicolumn{2}{|r|}{ Age: (Years) } & $53.91( \pm 14.95)$ \\
\hline \multicolumn{2}{|r|}{ Age at onset: (Years) } & $38.34( \pm 16.08)$ \\
\hline \multicolumn{2}{|c|}{ Positive Family History for Psychiatric Disorders } & $46.6 \%$ \\
\hline \multirow{5}{*}{ Primary Psychiatric Diagnoses } & Major Depressive Disorder & $49,1 \%$ \\
\hline & Bipolar Disorder & $15,7 \%$ \\
\hline & Generalized Anxiety Disorder & $5,5 \%$ \\
\hline & Adjustment Disorder & $5,5 \%$ \\
\hline & Others & $24,2 \%$ \\
\hline \multirow{5}{*}{ Psychiatric Comorbidity } & None & $60 \%$ \\
\hline & Anxiety Disorders & $15,8 \%$ \\
\hline & Personality Disorders & $9,1 \%$ \\
\hline & Mood Disorders & $4,8 \%$ \\
\hline & Others & $30,3 \%$ \\
\hline \multirow{5}{*}{ Medical Comorbidity } & None & $32 \%$ \\
\hline & Hypertension & $12.1 \%$ \\
\hline & Diabetes & $7.3 \%$ \\
\hline & Gastrointestinal Diseases & $7,3 \%$ \\
\hline & Others & $41,3 \%$ \\
\hline \multicolumn{2}{|c|}{ Duloxetine Daily Dosage } & $70 \mathrm{mg} /$ day $( \pm 28.6)$ \\
\hline \multicolumn{2}{|r|}{ Side effects } & $15 \%$ \\
\hline \multirow{4}{*}{ Drugs combined with Duloxetine } & SSRIs + Duloxetine & $9.1 \%$ \\
\hline & Mood stabilizers + Duloxetine & $11 \%$ \\
\hline & Atypical Antipsychotics + Duloxetine & $13.3 \%$ \\
\hline & Mood Stabilizers + Atypical Antipsychotics + Duloxetine & $15.1 \%$ \\
\hline
\end{tabular}

In terms of tolerability, no significant difference was found across groups when comparing the administration of Duloxetine in monotherapy vs polytherapy, according to the occurrence and nature of side-effects $\left(\chi^{2}=0.912, p=0.634\right.$, Phi $=0.075)$. In the same way, no significant difference in terms of side-effects was found when the sample was subdivided according to age ( $\leq$ or $>65$ years) (presence of side effects: $\chi^{2}=2.072, \mathrm{p}=0.355$, Phi $=0.113$; types of sideeffects: $\chi^{2}=8.564, p=0.286$, Phi $\left.=0.230\right)$ (Table 1).

\section{DISCUSSION}

Given that Duloxetine has been approved for the treatment of MDD, GAD, stress urinary incontinence, diabetic neuropathy and fibromyalgia [3], the present study agrees with international guidelines (i.e., Canadian Network for Mood and Anxiety Treatments -CANMAT-, World Federation of Societies of Biological Psychiatry -WFSBP-) [28, 29], except for a subgroup of patients with BD and current major depressive episode. With respect to $\mathrm{BD}$, even though recent studies have reported the induction of hypomania and rapid cyclic in bipolar patients treated with Duloxetine, even in association with other compounds [30], no manic switch was reported in the present sample. This result should likely be put in relation with the concomitant use of moodstabilizers and antipsychotic agents in bipolar patients.

With respect to medical comorbidities, Duloxetine should be avoided in patients with a creatinine clearance of $<30$ $\mathrm{mL} / \mathrm{min}$ and in patients with hepatic impairment [31]. Duloxetine has been associated with slight increase in blood pressure but not with clinically significant cardiovascular risks (e.g., increased heart rate or blood pressure) [32]. Duloxetine is generally considered safe and well tolerated in patients with comorbid diabetes [33]. Significant increase in body weight has not been generally reported with Duloxetine therapy and a recent study focusing on this aspect observed that Duloxetinetreated patients experienced weight loss after short-term treatment, followed by modest weight gain over longer-term treatment. Taken as a whole, the size of the weight changes observed in the above-mentioned instead of present study and other clinical trials [34] suggests that this compound is supposed to have minimal effects on weight for most patients. 
Given that the majority of patients of the present sample had a medical comorbidity, this study is consistent with literature about Duloxetine' tolerability and safety in presence of medical illness [35].

Mean dosage of Duloxetine was $70 \mathrm{mg} /$ day $( \pm 28.6)$, which is within the recommended range in order to achieve major efficacy according to international guidelines (60-120 $\mathrm{mg}$ /day) [36, 37]. From this perspective, a recent doubleblind randomized trial found no significant differences in terms of efficacy when comparing Duloxetine $60 \mathrm{mg} /$ day and $120 \mathrm{mg} /$ day in the treatment of severe depressive symptoms [38]. However, in cases of treatment resistance and/or multiple comorbidity, dosages above $60 \mathrm{mg} /$ day may be required [13].

Side effects were reported by $15 \%$ of the sample. Given that all reported symptoms were transient and mild, in the present study Duloxetine showed an overall favourable tolerability. The most frequent side-effects were sedation and gastrointestinal symptoms. From this perspective, the most important result of the present study is that Duloxetine appeared to be well-tolerated also when used in coadministration. In fact, the majority of the sample was taking the compound in polytherapy (68\%). Of note, no differences in terms of tolerability were found when comparing monoversus polytherapy. If, on one hand, positive tolerability in the polytherapy group might be read in light of a prior attitude to tolerate complex pharmacological regimens, on the other hand, data from the monotherapy group seem to support the specific tolerability of the molecule. Indeed, reported data are consistent with literature about Duloxetine' tolerability $[39,9]$. Other frequently observed side-effects, such as dry mouth, headache, dizziness and fatigues, were not experienced by our sample [4].

Recent studies have shown that Duloxetine is effective and well tolerated also in the elderly, with promising results on cognitive functions [39]. From this perspective, the present study is consistent with these data, given that approximately $1 / 4$ of the sample could aged between 65 and 85 years and no significant difference was be detected when comparing side effects according to age ( $<$ or $\geq 65$ years). A recent article on this topic found Duloxetine to be welltolerated and safe even for the elderly, although indirect comparisons suggested that older people were more likely to experience dry mouth and constipation [40]. In this specific perspective, however, no difference in terms of type of sideeffects was found in the present sample when comparing subjects $<65$ years with the elderly.

Given that Duloxetine is primarily metabolized by CYP1A2 and, in light of its moderate capacity to inhibit CYP2D6, caution should be used when co-administered with drugs that inhibit CYP1A2 (e.g.: amitriptyline, clomipramine, clozapine, imipramine) or drugs that are metabolized by CYP2D6 enzymes (e.g.: fluoxetine, paroxetine, venlafaxine, haloperidol, perfenazine, risperidone, tioridazine, desipramine, amitriptyline, clomipramine and nortriptyline) $[41,42]$. In the present study, Duloxetine was mostly used in association with other compounds (68\% of the sample), such as antidepressants, mood stabilizers and atypical antipsychotics and, even though among associated compounds there were some of the aforementioned molecules, no interaction in terms of tolerability was reported. Another appreciable result from the present study is the high rate of benzodiazepines co-administration (60\%). Even though benzodiazepines were in most cases administered before $\mathrm{Du}-$ loxetine, it is noteworthy to mention a recent study which indicated that Duloxetine may enhance the effects of benzodiazepines [42].

The present study has some limitations that need to be acknowledged. In particular, the retrospective collection of clinical information might have limited the reliability of some measures. In addition, the frequent administration of Duloxetine within different polytherapy regimens might represent a confounding factor in the evaluation of side effects, so that studies focused on the tolerability of Duloxetine within standardized treatments are required. Moreover, reported results may refer to patients seeking psychiatric treatment and may not be representative of the entire population of subjects affected by comorbid affective disorders. In fact, prospective randomized double-blind studies on this topic with more specific measures of effectiveness are warranted in order to confirm the present results. Finally, given that this was a naturalistic, open study and not a randomized controlled trial, caution should be taken when looking at some positive results.

In conclusion, Duloxetine was well tolerated in a large sample of patients with affective disorders and psychopathological and medical comorbidities, with only minor and transient side-effects both in mono- and polytherapy sub-groups; moreover, it was safely associated with other psychotropic drugs across different groups of age, including the elderly.

\section{DISCLOSURE}

The authors do not have any conflict of interest with the content of the present article. Dr Dell'Osso and Prof. Altamura have served in speakers bureau of Ely Lilly and Boehringer Ingelheim. The present study was not supported by any pharmaceutical financial support. The other authors do not report any conflict of interest with the content of the present article.

\section{CONFLICT OF INTEREST}

The authors confirms that this article content has no conflicts of interest.

\section{ACKNOWLEDGEMENT}

Declared none.

\section{REFERENCES}

[1] Dell'Osso B, Buoli M, Baldwin DS, Altamura AC. Serotonin norepinephrine reuptake inhibitors (SNRIs) in anxiety disorders: a comprehensive review of their clinical efficacy. Hum Psychopharmacol 2010; 25: 17-29.

[2] Sussman N. SNRIs versus SSRIs: mechanisms of action in treating depression and painful physical symptoms. Prim Care Companion J Clin Psychiatry 2003; 5: 19-26.

[3] Khan AY, Macaluso M. Duloxetine for the treatment of generalized anxiety disorder: a review. Neuropsychiatr Dis Treat 2009; 5: 23-31.

[4] Carter NJ, McCormack PL. Duloxetine. A review of its use in the treatment of generalized anxiety disorder. CNS Drugs 2009; 23: 523. 
[5] Lantz RJ, Gillespie TA, Rash TJ, et al. Metabolism, excretion and pharmacokinetics of duloxetine in healthy human subjects. Drug Metab Dispos 2003; 31: 1142-50.

[6] Kuo F, Gillespie TA, Kulanthaivel P, et al. Synthesis and biological activation of some known and putative duloxetine metabolites. Bioorg Med Chem Lett 2004; 14: 3481-6.

[7] Spina E, Santoro V, D'Arrigo C. Clinically relevant pharmacokinetic drug interactions with second-generation antidepressants: an update. Clin Ther 2008; 30: 1206-27.

[8] Bymaster FP, Dreshfield-Ahmad LJ, Threlkeld PG, et al. Comparative affinity of duloxetine and venlafaxine for serotonin and norepinephrine transporters in vitro and in vivo, human serotonin receptor subtypes, and other neuronal receptors. Neuropsychopharmacol 2001; 25: 871-80.

[9] Hudson JI, Wohlreich MM, Kajdasz DK, Mallinckrodt CH, Watkin JG, Martynov OV. Safety and tolerability of duloxetine in the treatment of major depressive disorder: analysis of pooled data from eight placebo-controlled clinical trials. Hum Psychopharmacol 2005; 20: 327-41.

[10] Park YM, Lee BH, Lee HJ, Kang SG. Cholestatic jaundice induced by duloxetine in a patient with major depressive disorder. Psychiatry Investig 2010; 7: 228-30.

[11] Rosso G, Rigardetto S, Bogetto F, Maina G. A randomized, singleblind, comparison of duloxetine with bupropion in the treatment of SSRI-resistant major depression. J Affect Disord 2012; 136: 172-6.

[12] Sheehan DV, Chokka PR, Granger RE, Walton RJ, Raskin J, Sagman D. Clinical and functional outcomes in patients with major depressive disorder and painful physical symptoms switched to treatment with duloxetine. Hum Psychopharmacol 2011; 26: 242-51.

[13] Pitchot W, Scantamburlo G, Ansseau M. Duloxetine in major depressed patients resistant to SSRIs and/or venlafaxine. Psychiatr Danub 2010; 22: S106-7.

[14] Kelin K, Berk M, Spann M J, et al. Duloxetine $60 \mathrm{mg}$ /day for the prevention of depressive recurrences: post hoc analyses from a recurrence prevention study. Int J Clin Pract 2010; 64: 719-26.

[15] Baldwin D, Woods R, Lawson R, Taylor D. Efficacy of drug treatments for generalised anxiety disorder: systematic review and meta-analysis. BMJ 2011; 342: d1199.

[16] Beesdo K, Hartford J, Russell J, Spann M, Ball S, Wittchen HU. The short term and long term effect of duloxetine on painful physical symptoms in patients with generalized anxiety disorder: Result from three clinical trials. J Anxiety Disord 2009; 23: 1064-71.

[17] Wang J, Liu X, Mullins CD. Treatment adherence and persistence with Duloxetine, Venlafaxine XR, and Escitalopram among patients with major depressive disorder and chronic pain-related diseases. Curr Med Res Opin 2011; 27: 1303-13.

[18] Serafini G, Pompili M, Del Casale A, et al. Duloxetine versus venlafaxine in the treatment of unipolar and bipolar depression. Clin Ther 2010; 161: 321-7.

[19] Wise E, Wiltse CG, Iosifescu DV, Sheridan M, Xu YY, Raskin J. The safety and tolerability of duloxetine in depressed elderly patients with and without medical comorbidity. Int J Clin Pract 2007; 61: 1283-93

[20] American Psychiatric Association. Diagnostic and statistical manual of mental disorders $4^{\text {th }}$ ed. Washington, DC: American Psychiatric Press 2000.

[21] First MB, Spitzer RL, Gibbon M, Williams JBW. Structured Clinical Interview for DSM-IV-TR Axis I Disorders, Research Version, Non-patient Edition (SCID-I/NP). New York: Biometrics Research, New York State Psychiatric Institute 2002.

[22] First MB, Spitzer RL, Gibbon M, Williams JBW, Benjamin LS. Structured Clinical Interview for DSM-IV Axis II Personality Disorders, (SCID-II). Washington, D.C.: American Psychiatric Press, Inc. 1997.
[23] Hamilton M. A Rating Scale for Depression, Department of Psychiatry, University of Leeds. J Neurol Neurosurg Psychiatry 1960; 23: 56-62.

[24] Hamilton M. The assessment of anxiety states by rating. Br J Med Psychol 1959; 32: 50-5.

[25] Montgomery SA, Asberg M. A new depression scale designed to be sensitive to change. Br J Psychiatry 1979; 134: 382-9.

[26] Young RC, Biggs JT, Ziegler VE, Meyer DA. A rating scale for mania: reliability, validity and sensitivity. Br J Psychiatry 1978; 133: 429-35.

[27] Guy W. Clinical Global Impressions: In ECDEU Assessment Manual for Psychopharmacology Revised DHEW Pub. (ADM). Rockville, MD: National Institute for Mental Health 1976; pp. 218 22.

[28] Sidney HK, Raymond WL, Sagar VP, Scott BP, Arun VR. Canadian Network for Mood and Anxiety Treatments (CANMAT) Clinical guidelines for the management of major depressive disorder in adults. J Affect Disord 2009; 117: S1-S2.

[29] Bandelow B, Zohar J, Hollander E, et al. WFSBP task force on treatment guidelines for anxiety, obsessive-compulsive disorder and post-traumatic stress disorder. W J Biol Psychiatry 2008; 9 : 248-312.

[30] Peritogiannis V, Antoniou K, Mouka V, Mavreas V, Hyphantis TN. Duloxetine-induced hypomania: case report and brief review of the literature on SNRIs-induced mood switching. J Psychopharmacol 2009; 23: 592-6.

[31] Westanmo AD, Gayken J, Haight R. Duloxetine: a balanced and selective norepinephrine- and serotonin-reuptake inhibitor. Am J Health Syst Pharm 2005; 62: 2481-90.

[32] Dugan SE, Fuller MA. Duloxetine: a dual reuptake inhibitor. Ann Pharmacother 2004; 38: 2078-85.

[33] Crucitti A, Zhang Q, Nilsson M, Brecht S, Yang CR, Wernicke J. Duloxetine treatment and glycemic controls in patients with diagnoses other than diabetic peripheral neuropathic pain: a metaanalysis. Curr Med Res Opin 2010; 26: 2579-88.

[34] Wise TN, Perahia DG, Pangallo BA, Losin WG, Wiltse CG. Effects of the antidepressant duloxetine on body weight: analyses of 10 clinical studies. Prim Care Companion J Clin Psychiatry 2006; 8: 269-78.

[35] Wise TN, Wiltse CG, Iosifescu DV, Sheridan M, Xu JY, Raskin J. The safety and tolerability of duloxetine in depressed elderly patients with and without medical comorbidity. Int J Clin Pract 2007; 61: 1283-93.

[36] Kornstein SG, Russell JM, Spann ME, Crits-Christoph P, Ball SG. Duloxetine in the treatment of generalized anxiety disorder. Expert Rev Neurother 2009; 9: 155-65.

[37] Frampton JE, Plosker GL. Duloxetine: a review of its use in the treatment of major depressive disorder. CNS Drugs 2007; 21: 581609.

[38] Brecht S, Desaiah D, Marechal ES, Santini AM, Podhorna J, Guelfi JD. Efficacy and safety of duloxetine $60 \mathrm{mg}$ and $120 \mathrm{mg}$ daily in patients hospitalized for severe depression: a double-blind randomized trial. J Clin Psychiatry 2010; 72: 1086-94.

[39] Tarolla E, Brugnoli R, Pacheri P. Duloxetine: clinical efficacy, safety and tolerability in treatment of major depression. Giron ital PsicoPat 2007; 13: 258-78.

[40] Dolder C, Nelson M, Stump A. Pharmacological and clinical profile of newer antidepressants: implications for the treatment of elderly patients. Drugs Aging 2010; 28: 625-40.

[41] Lobo ED, Bergstrom RF, Reddy S, et al. In vitro and in vivo evaluations of cytochrome P450 1A2 interactions with duloxetine. Clin Pharmacokinet 2008; 47: 191-202.

[42] Knadler MP, Lobo E, Chappell J, Bergstrom R. Duloxetine: clinical pharmacokinetics and drug interactions. Clin Pharmacokinet 2011; 50: 281-94.

Received: January 30, 2012

Revised: May 05, 2012

Accepted: June 04, 2012

(C) Dell'Osso et al.; Licensee Bentham Open .

This is an open access article licensed under the terms of the Creative Commons Attribution Non-Commercial License (http://creativecommons.org/licenses/by-nc/3.0/) which permits unrestricted, non-commercial use, distribution and reproduction in any medium, provided the work is properly cited. 